\title{
First Principles Calculation of Elastic Moduli of Early-Late Transition Metal Alloys
}

\author{
William Paul Huhn and Michael Widom \\ Department of Physics, Carnegie Mellon University, Pittsburgh, PA 15213 \\ Andrew M. Cheung and Gary J. Shiflet \\ Department of Materials Science and Engineering, \\ University of Virginia, Charlottesville, VA 22904 \\ S. Joseph Poon \\ Department of Physics, University of Virginia, Charlottesville, VA 22904 \\ John Lewandowski \\ Department of Materials Science and Engineering, \\ Case Western Reserve University, Cleveland, OH 44106
}

\begin{abstract}
Motivated by interest in the elastic properties of high strength amorphous metals, we examine the elastic properties of select crystalline phases. Using first principles methods, we calculate elastic moduli in various chemical systems containing transition metals, specifically early (Ta,W) and late $(\mathrm{Co}, \mathrm{Ni})$. Theoretically predicted alloy elastic properties are verified for Ni-Ta by comparison with experimental measurements using resonant ultrasound spectroscopy. Comparison of our computed elastic moduli with effective medium theories shows that alloying leads to enhancement of bulk moduli relative to averages of the pure elements, and considerable deviation of predicted and computed shear moduli. Specifically, we find an enhancement of bulk modulus relative to effective medium theory and propose a candidate system for high strength, ductile amorphous alloys. Trends in the elastic properties of chemical systems are analyzed using force constants, electronic densities of state and Crystal Overlap Hamilton Populations. We interpret our findings in terms of the electronic structure of the alloys.
\end{abstract}

\section{INTRODUCTION}

Elastic moduli are important for understanding various properties of amorphous metals. Bulk moduli, shear moduli, and their ratio correlate to glass transition temperature ${ }^{1,2}$, , glass forming ability $\underline{\underline{3}}$, brittleness $\underline{4}^{\underline{-6}}$, Grüneisen parameters ${ }^{7}$, maximum resolved shear stress at yielding ${ }^{8}$, chemical bonding type ${ }^{9}$, and possibly fragility $\underline{9}$. Knowledge of the bulk and shear moduli is thus important for materials design. However, amorphous materials commonly contain at least 4 chemical species, making exhaustive experimental evaluation of candidate materials impossible. Empirical methods for predicting stoichiometries with desired properties are therefore necessary.

First principles computational methods prove fruitful, owing to their chemical specificity and absence of adjustable parameters, as well as the insight they yield into electronic structure. However, amorphous metals pose computational difficulties, as they lack both spatial periodicity and a unique structure. While the first problem can be practically overcome by imposing suitably large periodic boundary conditions, this requires hundreds of atoms per computational cell, straining computational resources, and requiring averaging over multiple samples to remove sample dependence. The second problem can be partially overcome by running molecular dynamics on a liquid sample then rapidly quenching the sample. However the requirement for equilibration further increases the computational time necessary. Hence we adopt a dif- ferent strategy.

Frank Kasper phases ${ }^{10}-12$ are complex but otherwise ordinary crystalline phases. Due to their topological close packing they exhibit local icosahedral ordering similar to that found in many amorphous metals. Fig. 11 shows the standard Voronoi polyhedra of Frank-Kasper structures. We expect that the similar local chemical environments of the Frank Kasper phases can be used to mimic amorphous metals, yielding "amorphous approximants", similar in concept to "quasicrystal approximants". These crystalline phases will be used to understand trends in the elastic properties of amorphous metals. It is observed that in amorphous metals, shear moduli are typically 20\%-30\% lower, and bulk moduli 5\%-10\% lower, compared to crystalline phases of similar composition ${ }^{5}$. Many crystalline phases have small unit cells compared to system sizes required to reproduce amorphous structures.

Amorphous metals can exhibit high strength 13 , but often at the cost of a lack of ductility owing to the absence of dislocations. Designing amorphous alloys for high ductility is impeded both by the challenge of formulating accurate amorphous structural models, and by the lack of a valid predictive theory of ductility even for the case of crystalline compounds, though there are empirical rules based on the Poisson ratio, or equivalently the shear/bulk modulus ratio. We hypothesize that relatively ductile crystalline compounds will tend to create relatively ductile amorphous compounds. Further, metallic glass composites, consisting of crystalline grains embed- 


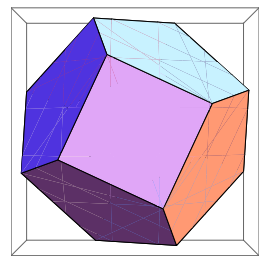

(a) $\mathrm{CN}=10,(0,2,8)$

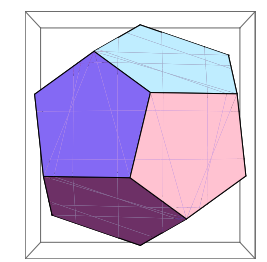

(b) $\mathrm{CN}=12,(0,0,12)$

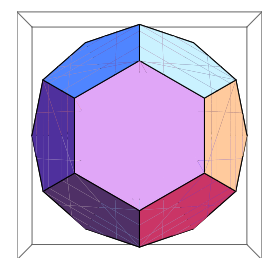

(c) $\mathrm{CN}=14,(0,0,12,2)$

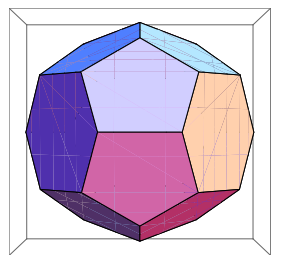

(d) $\mathrm{CN}=15,(0,0,12,3)$

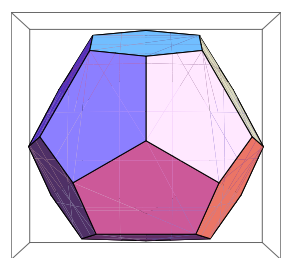

(e) $\mathrm{CN}=16,(0,0,12,4)$

FIG. 1: Frank-Kasper Voronoi polyhedra, listing coordination numbers and polyhedron codes.

ded in an amorphous matrix, have been shown to increase the toughness, impact resistance and plastic strain to failure $\stackrel{14}{\underline{14}}$, further motivating the investigation into elastic properties of crystalline phases in metallic glass-forming alloy systems.

\section{BACKGROUND AND METHODS}

\section{A. Elasticity}

The fundamental equation of linearized elasticity is $\sigma_{i}=C_{i j} \epsilon_{j}$, where $\sigma$ is the stress tensor, $\epsilon$ is the strain tensor, and $C$ the stiffness tensor relating the two. Here we employ Voigt notation, converting the tensors $\sigma$ and $\epsilon$ into vectors with 6 components $\{x x, y y, z z, x y, x z, y z\}$. For crystals $C$ depends on the symmetry of the material in question ${ }^{15}$ and contains at least three independent parameters. For the special case of isotropic materials $C$ has only two independent parameters, the bulk modulus $K$ and the shear modulus $G$. The non-vanishing elements are $C_{11}=C_{22}=C_{33}=K+4 G / 3, C_{12}=C_{13}=C_{23}=$ $K-2 G / 3$, and $C_{44}=C_{55}=C_{66}=G$. An additional elastic quantity of interest is the Poisson ratio $\nu$, defined to be the negative of the ratio of axial strain to transverse strain. For crystals $\nu$ depends on the direction of applied stress, but in the isotropic case it reduces to

$$
\nu=\frac{3-2 G / K}{6+2 G / K} .
$$

Notice that $\nu$ is a function of the shear to bulk modulus ratio $G / K$, also known as the Pugh ratio $\underline{16}$.

Ductility and Poisson's ratio are positively correlated in both polycrystalline $\frac{16}{\underline{6}}$ and amorphous $\underline{\underline{4}}$ solids, with $\nu \geq 0.32$ (equivalently $G / K \leq 0.41$ ) proposed as a empirical criterion for good ductility $\underline{17}$. Seeking simultaneously high strength and ductility, it is natural to choose chemical species which in elemental form have large $K$. The species chosen must also be known good glass formers. Early-late transition metal alloys fit both criteria, as the transition metals from Group IV to XI all have $K \geq 100 \mathrm{GPa}$, and they are one of the most frequently examined classes of amorphous metals, . Size mismatch criteria favor using transition metals from different rows and columns of the periodic table, e.g. Ta and $\mathrm{W}$ for early transition metals (ETMs), and Co and $\mathrm{Ni}$ as late transition metals (LTMs). Co and W are of particular interest for materials design as Co-based glasses exhibit ultrahigh fracture strength, W-based glasses have the highest known glass transition temperature for bulk metallic glasses, and both have high Young's modulus 9,18,19.

Ordinary crystalline materials contain randomly orientated microscopic grains and appear macroscopically isotropic. To compute the elastic properties for these "polycrystals", orientational averaging is required. Each grain is described microscopically by the same stiffness matrix, which contains between 3 (cubic) and 21 (triclinic) independent parameters ${ }^{20}$, but macroscopically the crystal is isotropic with only two independent parameters $K$ and $G$. In the Voigt average, the stiffness matrix $C$ is averaged over orientations 21 , which is exact if stress is uniform throughout space. In the Reuss average, the compliance matrix $S=C^{-1}$ is averaged over orientations, which is exact if strain is uniform throughout space. The Voigt average systematically overestimates the isotropic moduli, while the Reuss average systematically underestimates the moduli. Empirically, the arithmetic mean of the two, known as the Hill average ${ }^{22}$, gives improved agreement with experiment, and this is what we will report.

The polycrystalline average assumes the stiffness matrices of various grains are identical to the perfect crystal, differing only by relative orientation. To obtain macroscopic elastic moduli in materials where chemical environment spatially varies, in particular in materials containing chemically distinct grains, it is necessary to include effects arising from fluctuations in the local stiffness matrices. A general class of approximation schemes known as "effective medium theories" exists where each grain with its local stiffness matrix interacts with an effective medium characterized by a background stiffness matrix incorporating the interactions of all other grains. A popular effective medium theory is the coherent potential approximation (CPA $)^{23}$, a self-consistent formalism in which the background stiffness matrix is taken as the macroscopic average itself. The self-consistent interaction of grains yields a pair of coupled non-linear equations,

$$
\sum_{i} \phi_{i} \frac{K_{i}-K}{3 K_{i}+4 G}=0
$$


and

$$
\sum_{i} \phi_{i} \frac{G_{i}-G}{5 G(3 K+4 G)+6(K+2 G)\left(G_{i}-G\right)}=0,
$$

which can be solved numerically for the effective $K$ and $G$, where $\phi_{i}$ denotes the volume fraction of grain type $i$ in the material. $K_{i}$ and $G_{i}$ denote the bulk and shear modulus of grain type $i$, respectively.

Although intended for mixtures of crystalline grains, we will apply CPA in the limit where each grain shrinks to a single atom, to estimate the elastic moduli of compounds using the self-consistent average of properties of the constituent pure elements. Our usage of CPA may be viewed as a higher-order correction to the well-known but highly empirical "rule of mixtures" paradigm common in materials design, which has already been applied to bulk metallic glasses 24 . CPA takes into account the pure elemental properties but lacks information about interspecies bonding and specific alloy crystal structure. Thus we take CPA as a convenient, physically motivated interpolation to establish a baseline for comparison with the computed alloy moduli revealing the specific contributions of structure and bonding, though we note that metallic glasses exist where trace changes in composition yield relatively large changes in $\mathrm{K}$ and $\mathrm{G}$ due to alteration of chemical bonding type $\mathrm{2}^{25}$.

\section{B. First principles methods}

Our first principles calculations use the Vienna AbInitio Simulation Package (VASP) 26,27 , a plane wave abinitio package implementing PAW pseudopotentials 28 in the PW91 ${ }^{29}$ generalized gradient approximation to density functional theory (DFT). VASP calculates total energies, forces, elastic moduli, and electronic structure. All structures are relaxed until the maximum ionic force is below $0.01 \mathrm{eV} / \AA$, and the k-point density is subsequently increased until total energy per atom has converged to within $0.1 \mathrm{meV} /$ atom. Default plane wave energy cutoffs are used for total energy calculations. In structures containing $\mathrm{Co}$ and $\mathrm{Ni}$, spin polarization has been included. However, we do not include spin-orbit coupling despite the presence of $5 \mathrm{~d}$ elements Ta and $\mathrm{W}$. Total energies are converted to enthalpies of formation by subtracting from the tie-line joining the total energies of pure elements in their stable crystalline forms 30 .

We perform elastic calculations using a finite difference method internal to VASP. To ensure proper convergence of elastic moduli, we increase the k-point density until the all polycrystalline averages converge to within $2 \%$, then increase the energy cutoff until the polycrystalline averages converge. Convergence in energy cutoff occurs at $360 \mathrm{eV}$, with the exception of $\mathrm{Ni}_{2} \mathrm{Ta}$ and $\mathrm{Ni}_{4} \mathrm{~W}$ where 400 $\mathrm{eV}$ and $440 \mathrm{eV}$ respectively were required. All structures were tested for mechanical stability, and elastic constants were not calculated for structures that were found to be mechanically unstable, though they were included in the enthalpy of formation plots, as it is possible for the stabilizing distortions to affect the calculated total energy only weakly.

To quantify bond strength between individual atoms in structures, we calculate interatomic force constants and the Crystal Overlap Hamilton Population (COHP). To calculate the force constant $k_{\alpha \beta}$ between atoms $\alpha, \beta$ at position $\vec{r}_{\alpha, \beta}$, separated by a bond in the direction $\hat{\gamma}_{\alpha \beta}$, we calculate the Hessian matrix $\mathbf{H}_{\alpha \beta}=d^{2} E / d \delta \vec{r}_{\alpha} d \delta \vec{r}_{\beta}$. We use density functional perturbation theory internal to VASP to calculate $\mathbf{H}$ within a supercell of sufficient size that atoms lie at least $4.2 \AA$ away from their own repeated images. Restricting our attention to longitudinal (bond stretching) interactions and assuming central forces, we define

$$
k_{\alpha \beta} \equiv \hat{\gamma}_{\alpha \beta} \cdot \mathbf{H}_{\alpha \beta} \cdot \hat{\gamma}_{\alpha \beta}
$$

as the projection of the Hessian along $\hat{\gamma}_{\alpha \beta}$. $k_{\alpha \beta}$ must be positive for the force to be stabilizing.

The COHP provides an electronic structure-based characterization of interatomic bond strength ${ }^{31}$. One calculates matrix elements $\left\langle\alpha L|H| \beta L^{\prime}\right\rangle$ of the density functional theory Hamiltonian between localized atomic orbitals $L$ and $L^{\prime}$ on a pair of atoms $\alpha$ and $\beta$, then multiplies by the density of states $N_{\alpha L, \beta L^{\prime}}$ projected onto the two orbitals. We calculate wave functions using a TBLMTO method 32 then calculate an integrated COHP for a pair of atoms $\alpha \beta$, summing over atomic orbitals and integrating over energies up to the Fermi energy.

\section{Experimental Methods}

To check the validity of our first principles calculations we have prepared samples of single phase alloys of Ni-Ta by arc melting the pure element constituents ( $\mathrm{Ni} 99.995 \%$ and Ta 99.95\%) under an argon atmosphere. The alloys were then suction cast into water cooled copper molds to form rods of $2 \mathrm{~mm}$ diameter. Single phases of the rod samples were verified by x-ray powder diffraction (XRD) (Figure2). Cylindrical samples were obtained by sectioning the rods with a diamond saw into $4 \mathrm{~mm}$ segments. The ends of the segments were polished to a 3 micron finish. The elastic constants of the cylinders were calculated using data obtained from a Magnaflux Quasar Resonant Ultrasound Spectrometer. RUS involves placing the cylindrical samples diagonally between two ultrasonic transceivers and recording the natural modes of vibration $^{33}$. A Levenberg-Marquardt algorithm is then used to determine the elastic constants by finding the best fit solution through minimization of the difference between measured and calculated natural modes through iterative changes to the values of elastic constants. 


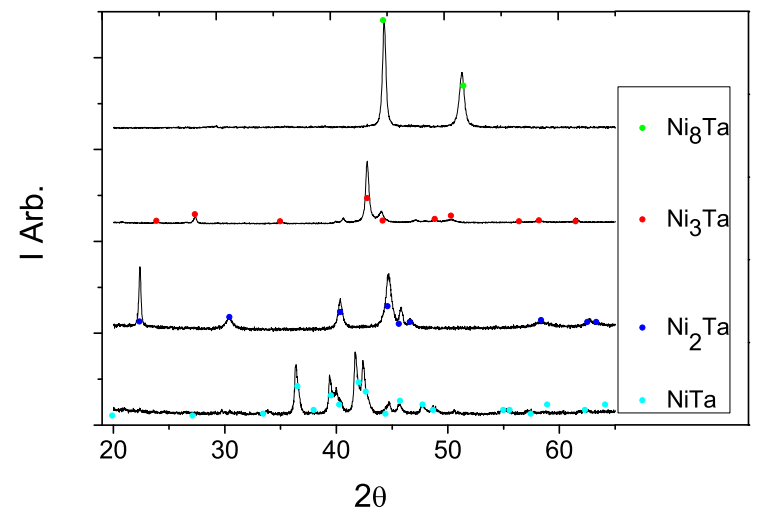

FIG. 2: XRD verification of single phases of $\mathrm{Ni}_{8} \mathrm{Ta}$, $\mathrm{Ni}_{3} \mathrm{Ta}, \mathrm{Ni}_{2} \mathrm{Ta}$, and NiTa.

The RUS measurement technique is limited to nonmagnetic samples. The study using the RUS measurements for comparison to theory is limited to the Ni-Ta system where single phases of $\mathrm{Ni}_{8} \mathrm{Ta}, \mathrm{Ni}_{3} \mathrm{Ta}, \mathrm{Ni}_{2} \mathrm{Ta}$ and $\mathrm{NiTa}$, which are nonmagnetic, can be produced, allowing for RUS measurements. Rods of the pure elements Ni and Ta can also be cast, but of the two only Ta produces nonmagnetic rods.

\section{RESULTS}

In this section we first discuss atomistic structure, then present results on thermodynamic stability for each alloy system considered, finally we address the elastic moduli.

\section{A. Structure}

Because we consider a large number of specific structures, we establish a numbering scheme to unambiguously identify them (see Table I). In the text we refer to a given crystalline material using the notation Compound.Pearson (e.g. $\mathrm{Co}_{2}$ Ta.cF24). Of particular interest are the Frank-Kasper structures, which we take as amorphous approximants owing to the prevalence of their coordination polyhedra in many metallic glasses ${ }^{34.35}$. Canonical Frank-Kasper polyhedra have coordination numbers $\mathrm{CN}=12,14,15$ and 16 . However, we include the Bernal holes 36.37 , notably $\mathrm{CN}=10$, as tetrahedral but non-canonical Frank-Kasper polyhedra ${ }^{34}$ suitable for smaller atoms. Also, the tetrahedral closepacking of the Frank Kasper phases matches the packing properties expected in bulk metallic glasses 38 .

To justify use of crystalline phases as amorphous approximants, we compare Honeycutt-Andersen common neighbor statistics $\frac{39,40}{}$, of crystalline and amorphous structures. Amorphous structures were simulated by quenching 100 atom liquid structures in NPT ensembles from $\mathrm{T}=2500 \mathrm{~K}$ down to $300 \mathrm{~K}$ over runs of more

\begin{tabular}{r|rrr|l|l} 
Ref. & Prototype & Strukt. Pearson & FK? & CN \\
1 & $\mathrm{Cu}$ & $\mathrm{A} 1$ & $\mathrm{cF} 4$ & $\mathrm{NA}$ & 12 \\
2 & $\mathrm{~W}$ & $\mathrm{~A} 2$ & $\mathrm{cI} 2$ & $\mathrm{NA}$ & 14 \\
3 & $\mathrm{Mg}$ & $\mathrm{A} 3$ & $\mathrm{hP} 2$ & $\mathrm{NA}$ & 12 \\
4 & $\mathrm{Fe}_{6} \mathrm{~W}_{7}$ & $\mathrm{D} 8_{5}$ & $\mathrm{hR} 13$ & $\mu$ & $12,14,15,16$ \\
5 & $\mathrm{Al}_{2} \mathrm{Cu}$ & $\mathrm{C}_{16}$ & $\mathrm{tI} 12$ & non-FK & 10,15 \\
6 & $\mathrm{AuCu}_{3}$ & $\mathrm{~L} 1_{2}$ & $\mathrm{cP} 4$ & $\mathrm{NA}$ & 12,18 \\
7 & $\mathrm{BaPb}_{3}$ & & $\mathrm{hR} 12$ & $\mathrm{NA}$ & 12,18 \\
8 & $\mathrm{MgNi}_{2}$ & $\mathrm{C} 36$ & $\mathrm{hP} 24$ & Laves & 12,16 \\
9 & $\mathrm{MgZn}_{2}$ & $\mathrm{C} 14$ & $\mathrm{hP} 12$ & Laves & 12,16 \\
10 & $\mathrm{MgCu}_{2}$ & $\mathrm{C} 15$ & $\mathrm{cF} 24$ & Laves & 12,16 \\
11 & $\mathrm{Ni}_{3} \mathrm{Sn}$ & $\mathrm{D} 0_{19}$ & $\mathrm{hP} 8$ & $\mathrm{NA}$ & 12,18 \\
12 & $\mathrm{Al}_{3} \mathrm{Ti}$ & $\mathrm{D} 0_{22}$ & $\mathrm{tI} 8$ & $\mathrm{NA}$ & $12,14,16$ \\
13 & $\mathrm{Cu}_{3} \mathrm{Ti}$ & $\mathrm{D} 0_{8}$ & $\mathrm{oP} 8$ & $\mathrm{NA}$ & $12,14,16$ \\
14 & $\mathrm{NbPt}_{3}$ & & $\mathrm{mP} 16$ & $\mathrm{NA}$ & $12,14,16$ \\
15 & $\mathrm{MoSi}_{2}$ & $\mathrm{C} 11_{b}$ & $\mathrm{tI} 6$ & $\mathrm{NA}$ & 14 \\
16 & $\mathrm{MoNi}_{4}$ & $\mathrm{DA} 1_{a}$ & $\mathrm{tI} 10$ & $\mathrm{NA}$ & 13,14 \\
17 & $\mathrm{Pt}_{8} \mathrm{Ti}$ & & $\mathrm{tI} 18$ & $\mathrm{NA}$ & 13,14 \\
18 & $\mathrm{MoNi}$ & & $\mathrm{oP} 56$ & $\delta$ & $12,14,15,16$
\end{tabular}

TABLE I: Structure types considered listing common prototype, and Strukturbericht and Pearson notations. Frank-Kasper phases list common names. * indicates symmetry-breaking distortion. "Non-FK" indicates non-canonical Frank-Kasper phase containing $\mathrm{CN}=10$

Bernal Hole. The final column contains a list of coordination numbers from Voronoi polyhedra appearing in the structure $e^{34.36 .37}$.

than 15ps. All simulations were performed at the gamma point with default energy cutoffs. Shown in Figure 3 is the number of common neighbors between two bonded atoms of given types. We show results for $\mathrm{Co}-\mathrm{Ta}$, but $\mathrm{Ni}-\mathrm{Ta}, \mathrm{Ni}-\mathrm{W}$, and $\mathrm{Co}-\mathrm{W}$ were also simulated and generated similar results, with the exception of one Ni-W amorphous sample which was likely out of equilibrium.

All structures have many bonds with $n=5$ common neighbors, especially between unlike atomic species, reflecting the prevalence of icosahedral ordering in FrankKasper phases and amorphous materials. Very few CoCo bonds have $n=6$ common neighbors, and very few Ta-Ta bonds have $n=4$ common neighbors, reflecting the relative sizes of Co and Ta atoms. Because hR13 is a canonical Frank-Kasper phase with no bonds sharing $n=4$ common neighbors, we utilize tI12 to capture the role of $n=4$ Co-Co bonds.

\section{B. Stability}

Figure 4 summarizes our calculated enthalpies of formation. Vertices of the convex hull of enthalpy as a function of composition are predicted to be stable phases at low temperature ${ }^{30}$. We employ special plotting symbols to indicate phases claimed experimentally to be stable at low temperature (heavy circles) and high temperature (light circles). Phases whose stability or existence is in question are shown as squares. From the prevalence of heavy circles on or near the convex hull we see general 


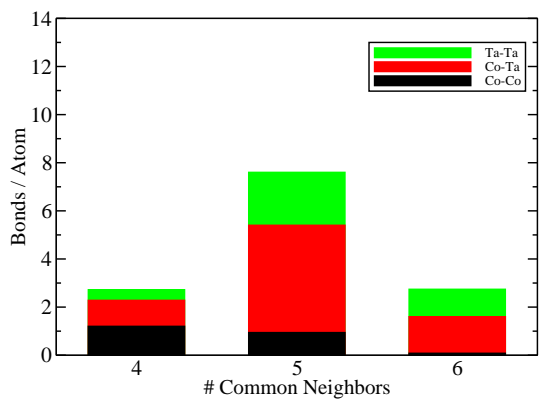

(a) Amorphous $\mathrm{Co}_{50} \mathrm{Ta}_{50}$

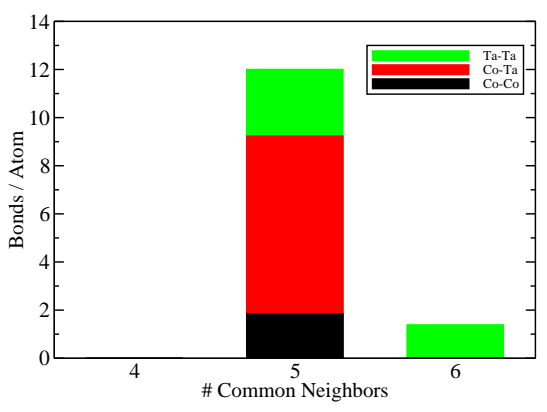

(b) $\mathrm{Co}_{6} \mathrm{Ta}_{7} \cdot \mathrm{hR} 13$

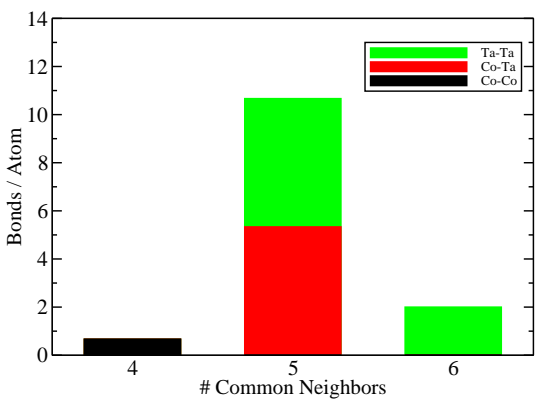

(c) $\mathrm{Co}_{2}$ Ta.tI12

FIG. 3: Stacked bar chart of Honeycutt-Andersen common neighbor statistics ${ }^{39,40}$.

(though imperfect) agreement with the experimentally reported phase diagrams. We briefly summarize our findings for the four alloy systems of primary interest.

\section{Co-Ta}

For Co-Ta (Fig. 4a), at $x=0.25$, we find that $\mathrm{cP} 4$ and hR12 (reference numbers 6 and 7 ) are nearly degenerate, with $\mathrm{cP} 4$ (stability not reported experimentally) favored by $1 \mathrm{meV} /$ atom, which is closer than DFT can reliably distinguish. At $x=0.33$, three different Laves phases have been reported (reference numbers 8-10), with conflicting claims of stability and uncertain composition. We find that none of these phases lies on the convex hull. Further, all of their structures are mechanically unstable to deformation, casting doubt on the reported structure and stability of this phase. In our plot, we show the energy of a distorted cF24 structure which is mechanically stable, for comparison with the undistorted hP12 and hP24. In the vicinity of $x=0.5$ lies the Frank-Kasper $\mu$ phase. This phase is common to many alloy systems containing fourth and fifth row early transition metals with third row late transition metals. In most cases the phase shows a broad composition range at high temperature but favors an ETM-rich low temperature limit (i.e. with the mixed occupancy $3 a$ site occupied by an ETM). This feature is correctly reproduced by our calculation. At $x=0.67$, the tI12 phase (reference number 5 ) is a noncanonical Frank-Kasper phase, as it contains a $\mathrm{CN}=10$ Bernal Hole, in addition to a canonical $\mathrm{CN}=15$ Kasper polyhedron.

\section{2. $\mathrm{Ni}-\mathrm{Ta}$}

For Ni-Ta (Figure 4b) at $x=0.11$ we find $\mathrm{Ni}_{8} \mathrm{Ta} . \mathrm{tI} 18$ to be low-temperature stable. The experimental phase diagram has a tI36 structure stable, however no crystallographic refinement exists, so we use the known tI18 phase instead. There is disagreement on recent phase diagrams concerning the stable structure at $x=0.25$, and we find three different structures have nearly identical enthalpies (tI8 is the lowest). The main source of disagreement between our $\mathrm{T}=0 \mathrm{~K}$ phase diagram and experimental phase diagrams is the stability of NiTa.hR13, with even the ETM-rich variant lying $7 \mathrm{meV} /$ atom above the convex hull. Fig. 2 shows the diffraction patterns of our experimentally cast rods, verifying the existence of the expected phases in our own samples.

\section{3. (Co,Ni)-W}

Our calculations verify the known $\mathrm{Co}_{3} \mathrm{~W}$ and $\mathrm{Ni}_{3} \mathrm{~W}$ phase stabilities. However, the reported $\mathrm{Co}_{7} \mathrm{~W}_{6}$ phase lies above the convex hull, and additionally favors the ETM-rich limit contrary to experimental report. This phase has not been reported in the Ni-W alloy system, and we indeed find it lies well above the convex hull. However, we will study the electronic and elastic properties of this hypothetical phase in order to elucidate trends with respect to composition. Notice that enthalpies of formation for alloys with $\mathrm{W}$ are lower than enthalpies with Ta. This does not necessarily reflect lower mechanical stability or melting points for the compounds, as the greater cohesive energy of tungsten compared to tantalum contributes to a reduction of the alloy formation enthalpies. An equiatomic Ni-W phase with orthorhombic symmetry has been observed low temperature stable $\stackrel{41}{ }$, however no atomic structural data exists. Owing to similar chemical identity and Bravais lattice, we attempted to use the Frank-Kasper phase MoNi.oP56 with Mo substituted for W, but found that this structure lies $66 \mathrm{meV} /$ atom above the convex hull and likely is not the correct phase. It was also too computationally expensive to compute elastic moduli for this phase.

\section{Binary Elastic Moduli}

We examine the effect of alloying on the elastic moduli by using CPA to approximate a hypothetical alloy where no interspecies interactions exist. That is, for a well-ordered phase with stoichiometry $\mathrm{A}_{\mathrm{x}} \mathrm{B}_{1-\mathrm{x}}$, we compare its elastic moduli to a hypothetical solution of pure 


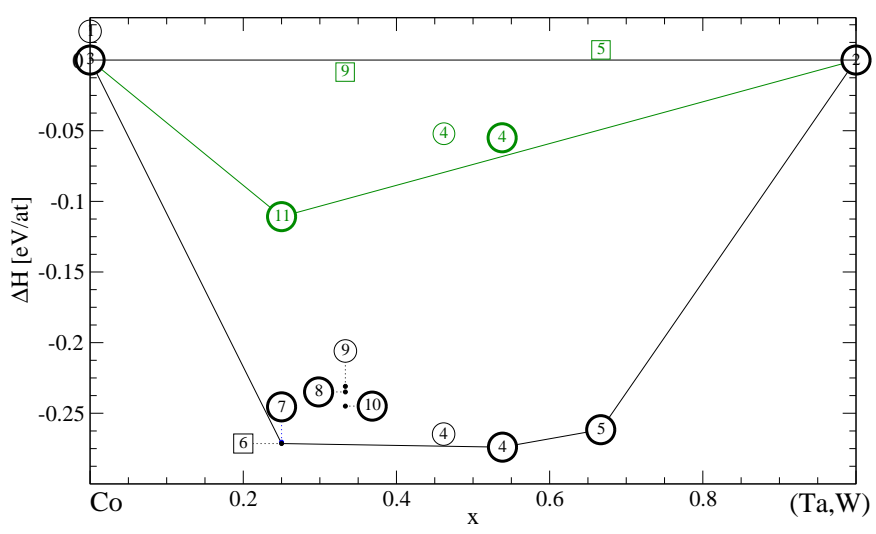

(a) Co- $(\mathrm{Ta}, \mathrm{W})$

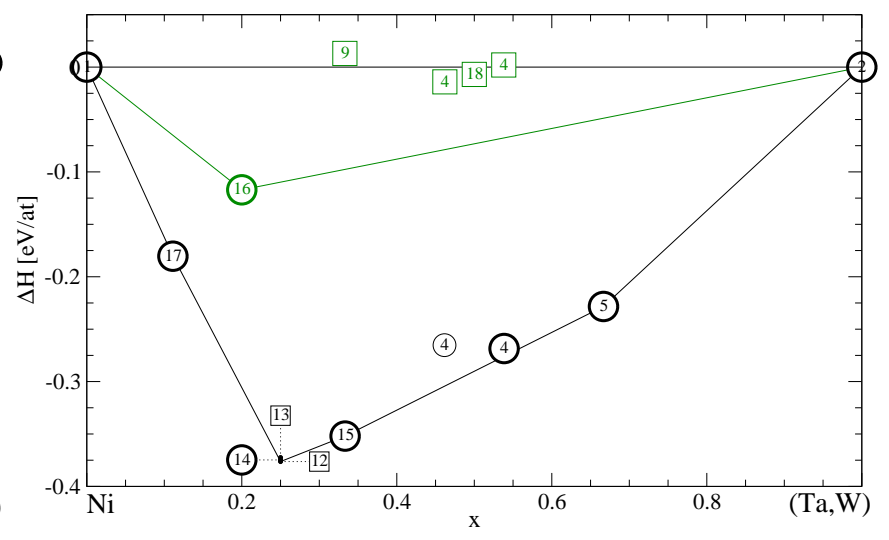

(b) Ni-(Ta,W)

FIG. 4: Enthalpies of formation for $(\mathrm{Co}, \mathrm{Ni})-(\mathrm{Ta}, \mathrm{W})$ alloys. Values for Ta shown in black, for $\mathrm{W}$ shown in green. Plotting symbols explained in text.

specie $\mathrm{A}$ and pure specie $\mathrm{B}$ with a stoichiometric ratio $\mathrm{x}:(1-\mathrm{x})$ in the CPA approximation. As input for CPA, we use our computed elemental elastic moduli. These agree closely with experimental moduli for Ta and $\mathrm{W}$ but are relatively high for Co and Ni. Note that our calculation are valid at $0 \mathrm{~K}$, while the experimental values are room temperature, so it is expected that our values should be high, especially for non-refractory elements. As the CPA approximation only incorporates elemental elastic moduli with no atomic environmental details, the deviation of computed polycrystalline moduli from the CPA approximation yields a measure of the relative importance of atomic environment and alloying species for elastic moduli.

All crystal structures are elastically anisotropic, and it is of interest to characterize the anisotropy of our amorphous approximants. We define three anisotropies ${ }^{20.42}$ : $A_{Z}$ (Zener), $A_{G}$ (shear) and $A_{E}$ (Young's) as

$$
\begin{gathered}
A_{Z}=\frac{2 C_{44}}{C_{11}-C_{12}}, \\
A_{G}=\frac{S_{44}+S_{66}}{2 S_{44}},
\end{gathered}
$$

and

$$
A_{E}=\frac{S_{11}}{S_{33}},
$$

all of which are 1 for isotropic structures, where $S_{i j}$ are elements of the compliance matrix. Table $\Pi$ compares calculated anisotropies of our hR13 and tI12 structures with the four pure elements. Our anisotropies are close to one, similar to those seen in the pure metals, with hR13 exhibiting less anisotropy than tI12. These anisotropies can be taken as estimates of the local anisotropy expected at the atomic level in actual amorphous structures. Recall that the polycrystalline averages are expected to reflect

\begin{tabular}{c|c|c|c|c} 
Composition & Structure & $A_{Z}$ & $A_{G}$ & $A_{E}$ \\
\hline Co-Ta & hR13 & 0.70 & 0.85 & 1.13 \\
Ni-Ta & hR13 & 0.81 & 0.91 & 1.14 \\
Co-W & hR13 & 0.76 & 0.88 & 1.18 \\
Ni-W & hR13 & 1.12 & 1.06 & 1.45 \\
\hline Co-Ta & tI12 & 0.90 & 1.17 & 1.27 \\
Ni-Ta & tI12 & 1.32 & 1.24 & 1.50 \\
Co-W & tI12 & 1.24 & 1.12 & 1.20 \\
Ni-W & tI12 & 1.42 & 1.16 & 1.07 \\
\hline Co & hP2 & 1.01 & 1.00 & 1.31 \\
Ni & cF4 & 2.18 & 1 & 1 \\
Ta & cI2 & 1.16 & 1 & 1 \\
W & cI2 & 0.83 & 1 & 1
\end{tabular}

TABLE II: Measures of anisotropy for materials of interest. $A_{G}=A_{E}=1$ for cubic structures.

the globally isotropic properties of the bulk amorphous structures.

Shown in Figure 5 are our calculated $\mathrm{K}$ values, compared with CPA estimates. All CPA estimates are indicated by lines, all calculated moduli by individual data points, and for Ni-Ta asterisks indicate experimental results. Our calculated Ni-Ta bulk moduli show excellent agreement with our experimental results. For all four chemical families, CPA gives reasonable estimates for bulk moduli, with at most a $16 \%$ deviation between estimated and calculated bulk moduli. However, in all alloy systems and for all structures examined, CPA underestimates the bulk modulus. This suggests the dominant correction to the bulk moduli is chemical bonding and not atomic environmental details such as the prevalence of tetrahedra. Shear moduli show relatively larger and less regular deviations from CPA, suggesting that bond topology plays a significant role. Nonetheless, our calculated Ni-Ta shear moduli are in good agreement with experiment (crosses).

Shown in Table III are the correlation coefficient for various linear regressions across all calculated CoTa, 


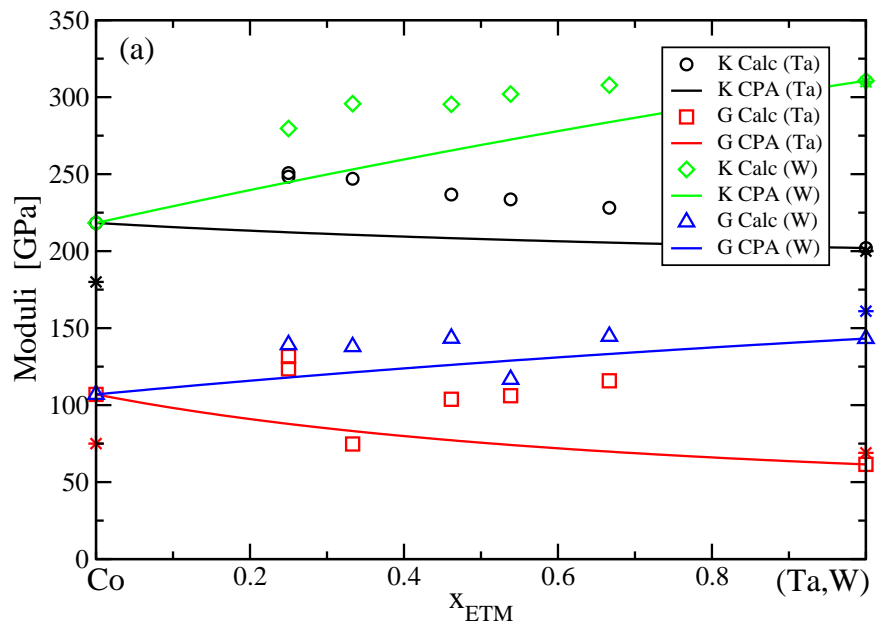

(a) Co-Ta

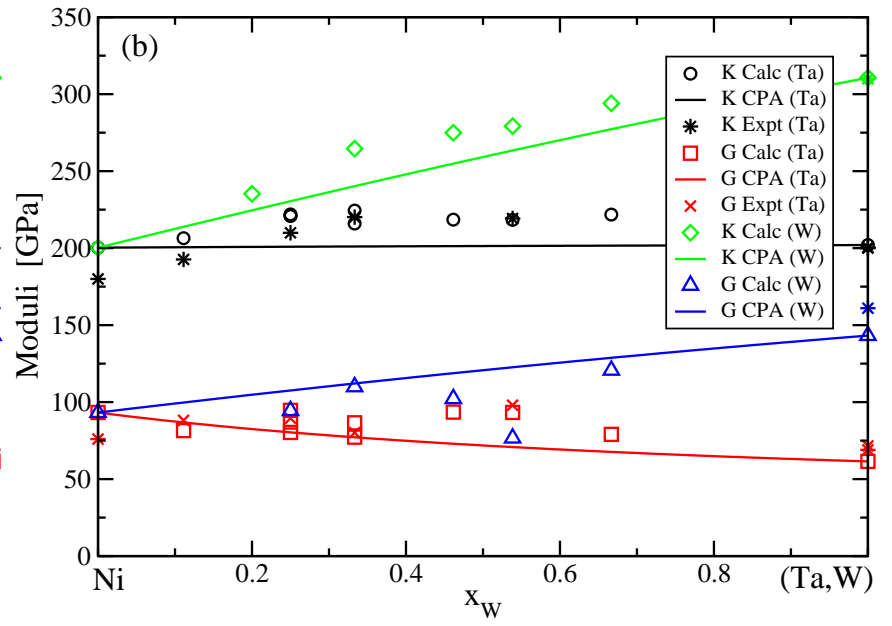

(b) Ni-Ta

FIG. 5: Elastic moduli for (Co,Ni)-(Ta,W) compounds. Lines show CPA estimates. Data points are our DFT calculations. Asterisks are experimental values from our experiments on Ni-Ta alloys and from the literature for pure elements.

\begin{tabular}{|c|c|c|c|c|}
\hline & $\Delta \mathrm{v}$ & $\Delta \mathrm{h}$ & $\Delta \mathrm{K}$ & $\Delta \mathrm{G}$ \\
\hline$\Delta \mathrm{v}$ & & 0.20 & -0.83 & -0.66 \\
\hline$\Delta \mathrm{h}$ & 0.20 & & 0.06 & -0.47 \\
\hline$\Delta \mathrm{K}$ & -0.83 & 0.06 & & 0.60 \\
\hline$\Delta \mathrm{G}$ & -0.66 & -0.47 & 0.60 & \\
\hline
\end{tabular}

TABLE III: Correlation coefficients for linear regressions involving elastic moduli and thermodynamic quantities of interest, where rows denote independent variables and columns dependent variables for a given regression.

CoW, NiTa, and NiW alloys, where the sign of the correlation coefficient denotes the sign of the slope. Here $\Delta \mathrm{K}$ and $\Delta \mathrm{G}$ (units $\mathrm{GPa}$ ) are deviations of calculated bulk and shear moduli from CPA estimates, $\Delta \mathrm{v}$ (units $\AA^{3}$ per atom) the deviation of volume per atom from a linear interpolation of pure elements, and $\Delta \mathrm{h}$ (units $\mathrm{eV}$ per atom) the enthalpy of formation per atom as illustrated in Fig. 4. Correlations of elastic moduli with $\Delta \mathrm{v}$ and $\Delta \mathrm{h}$ reflect structure and bonding effects that are missing from CPA. There is only a weak correlation between $\Delta \mathrm{h}$ and $\Delta \mathrm{v}$, though the associated slope is positive, expected as increased bond strength (more negative $\Delta \mathrm{h}$ ) draws atoms closer together (more negative $\Delta \mathrm{v}$ ). $\Delta \mathrm{G}$ and $\Delta \mathrm{K}$ are both correlated to $\Delta \mathrm{v}$, with $\Delta \mathrm{K}$ in particular strongly correlated. This is line with other work that shows that $\mathrm{K}$ and $\mathrm{G}$ correlate with $\mathrm{V}$ and that the correlation for $\mathrm{K}$ is particularly strong $\stackrel{9}{ }$. This is also true for individual chemical families, and for $\Delta \mathrm{K}$ all chemical families' regressions have similar slopes. This strong correlation of $\Delta \mathrm{K}$ with $\Delta \mathrm{v}$ explains why all structures have CPA underestimating the bulk moduli (positive $\Delta \mathrm{K}$ ), as all structures were also observed to have negative $\Delta \mathrm{v}$, as expected. The slopes of $\Delta \mathrm{G}$ and $\Delta \mathrm{K}$ are both negative, as decreasing $\Delta \mathrm{v}$ draws atoms closer together, shortening bonds and enhancing interatomic force constants. The observed correlation of $\Delta \mathrm{G}$ with $\Delta \mathrm{K}$ is likely due to the underlying correlation of each with $\Delta \mathrm{v}$.

A goal of metallic glass design is to predict glassforming compounds with high ductility. Thus, as a guide, we plot the Poisson ratio's of the various alloys under discussion. Our computed $\mathrm{T}=0 \mathrm{~K}$ crystalline Poisson ratios are expected to be systematically low relative to the corresponding glasses, as $\mathrm{G}$ decreases more rapidly than $\mathrm{K}$ as temperature increases $\frac{43}{}$, and amorphous $\mathrm{G}$ and $\mathrm{K}$ are lower relative to crystalline values by around $30 \%$ and $10 \%$, respectively. Here we see no systematic trend in the choice of Ta versus W (empty versus filled plotting symbols) but Ni generally has higher Poisson's ratio than Co (red versus blue). Empirically, it has been observed that $\nu=0.32$ serves as a rough criterion for separating ductile and brittle behavior in amorphous materials ${ }^{6}$. The majority of our Ni alloys lie above this criterion and Co alloy below. In particular, all $\mathrm{Ni}-\mathrm{W}$ alloys satisfy this criterion, and $\mathrm{Ni}-\mathrm{W}$ in the amorphous approximant structure hR13 shows particularly large Poisson's ratios. Combined with the large bulk modulus due to the presence of tungsten, we propose that $\mathrm{Ni}-\mathrm{W}$ is a candidate system for future research into strong amorphous materials with high ductility.

\section{ANALYSIS AND DISCUSSION}

To understand trends in elastic constants of these alloys we now look into the interatomic interactions. Within a first principles approach there is no unique decomposition of interactions into pairwise and manybody forces, and no simple notion of a chemical bond, especially for metals. However some heuristic measures 


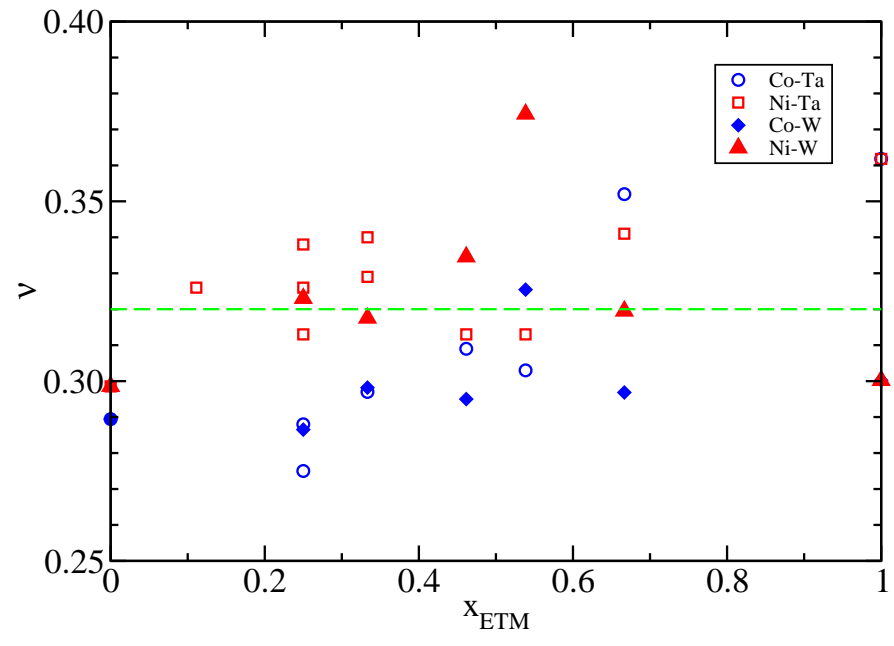

FIG. 6: Calculated Poisson ratios. Dashed line at $\nu=0.32$ is putative threshold for ductility.

are available. Here we examine the interatomic force constants, which can be regarded as springs connecting the atoms, and the crystal overlap Hamilton populations (COHPs) which are a measure of the covalency of electronic wave functions.

\section{A. Force Constants and COHP}

To compare different ETM and LTM substitutions, Table IV shows $\mathrm{k}$, the mean force constants for the near neighbor bonds of a given species combination, $\rho_{\mathrm{COHP}}$, the total iCOHP per unit volume for bonds of a given species combination up to $4 \AA$, and $\mathrm{K}$, the bulk moduli in the structural prototype tI12. To calculate the mean force constant, we sum force constants for all bonds up to $4 \AA$ for a given cell then divide by the number of atoms.

Both the mean force constant and $\rho_{\mathrm{COHP}}$ correlate with the bulk moduli. This is especially notable in the mean force constant, where there is a large increase in mean force constant performing a $\mathrm{Ta} \rightarrow \mathrm{W}$ substitution and a relatively small increase performing a $\mathrm{Ni} \rightarrow \mathrm{Co}$ substitution, but the effect is also present in $\rho_{\mathrm{COHP}}$. As a force constant gives a measure of the stiffness of an individual bond, this mean force constant gives a measure of the total stiffness of all bonds, and bulk modulus is increased under chemical substitution by an overall increase in the interatomic force constant. We also see in Table IV that performing a $\mathrm{Ni} \rightarrow \mathrm{Co}$ or a $\mathrm{Ta} \rightarrow \mathrm{W}$ substitution enhances $\rho_{\mathrm{COHP}}$. Thus these substitutions have enhanced the bonding nature of the electronic states.

To further understand the enhancement of bonding, we calculate electronic densities of state (Fig. 7). The low-energy peak near -4 or $-5 \mathrm{eV}$ consists of $s d$-hybrid orbitals, followed by a series of higher-energy peaks consisting solely of $d$ orbitals, with the Fermi level lying in the middle of the ETM $d$-band and at or above the top of the LTM $d$-band. For Co-W, the Co and $\mathrm{W} d$ bands are closely aligned, inducing strong hybridization of Co and $\mathrm{W} d$ orbitals. This effect is present in all structures we have examined. Performing a $\mathrm{W} \rightarrow$ Ta substitution shifts the ETM $d$-band up relative to the LTM $d$-band, decreasing the $d$-band overlap and diminishing hybridization. Performing a $\mathrm{Co} \rightarrow \mathrm{Ni}$ substitution shifts the LTM $d$-band down relative to the ETM $d$-band, also decreasing the $d$-band overlap. Both of these induce an decrease in the hybridization of the ETM-LTM $d$-bands. As hybridization generally creates bonding states below the Fermi level, this reduction in hybridization going from $\mathrm{W}$ $\rightarrow \mathrm{Ta}$ and $\mathrm{Co} \rightarrow \mathrm{Ni}$ decreases the overall bonding characteristic of the occupied states, leading to the observed trends in $\rho_{\mathrm{COHP}}$, and hence in bulk modulus.

\section{B. Microstructural Details: Ternaries and Quaternaries}

Elemental properties provide the dominant contribution to the elasticity of these ETM-LTM intermetallic compounds, as can be seen in the qualitative agreement of calculated alloy moduli with the CPA predictions shown in Fig. 5, In Table $\mathrm{V}$ we see that the small decreases in modulus from $\mathrm{Co}$ to $\mathrm{Ni}$, and the large increases from $\mathrm{Ta}$ to $\mathrm{W}$, are echoed in the moduli of the hR13 Frank-Kasper structure.

While binary amorphous metals exist, size mismatch criteria and material property tuning favor the usage of multiple constituent species in amorphous metals for practical applications, and thus the question of transferability of binary results to structures with 3 or more constituent species must be addressed. In addition, there is still the lingering need to quantify how atomic environment affects the elastic moduli. To answer both these questions, we perform chemical substitutions in a binary structure to yields ternaries and quaternary structures. This changes the chemical identities of formerly equivalent sites, altering local chemical ordering.

Shown in Table VI is a comparison of binary hR13 structures (including also alloys with $\mathrm{Nb}$, an ETM) with nearly equiatomic composition quaternary variants of $\mathrm{hR} 13$ and six associated ternaries. Site occupancies in the quaternary has been chosen to maintain the ETM/LTM nature of sites and minimize energy, and the ternaries were formed by keeping the early/late site identity fixed.

To compare our binary results to ternaries and quaternaries, we here use a simple chemical environment averaging scheme between ETM and LTM, with a equiatomic ABCD mixture with A and B LTM and C and D ETM approximated by $1 / 4^{*}(\mathrm{AC}+\mathrm{AD}+\mathrm{BC}+\mathrm{BD})$, and an $\mathrm{ABC}$ mixture (with $\mathrm{C}$ having near $50 \%$ concentration) approximated by $1 / 2 *(\mathrm{AC}+\mathrm{BC})$. Here $\mathrm{AC}, \mathrm{AD}, \mathrm{BC}$ and $\mathrm{BD}$ refer to the relevant binary $\mathrm{hR} 13$ structure with the associated chemical formula. As an example, the predicted bulk moduli of $\mathrm{Co}_{6} \mathrm{Ta}_{3} \mathrm{~W}_{4}$ would be the average bulk modulus of $\mathrm{Co}_{6} \mathrm{Ta}_{7}$ and $\mathrm{Co}_{6} \mathrm{~W}_{7}$. While this ignores interspecies ETM-ETM and LTM-LTM bonds present 


\begin{tabular}{|c|c|c|c|c|c|c|c|c|c|c|}
\hline Compound & ETM-E & $\Gamma \mathrm{M}(44 \mathrm{x})$ & ETM-L & TM $(32 \mathrm{x})$ & LTN & I-LT & $M(4 x)$ & mean & mean & \\
\hline $\mathrm{tI} 12$ & $\mathrm{R}$ & $\rho_{\mathrm{COHP}}$ & $\mathrm{R}$ & $\rho_{\mathrm{COHP}}$ & & $\mathrm{k}$ & $\rho_{\mathrm{COHP}}$ & $\mathrm{k}$ & $\rho_{\mathrm{COHP}}$ & K \\
\hline $\mathrm{NiTa}_{2}$ & 3.124 .2 & 0.31 & 2.634 .7 & 0.25 & 2.43 & $\overline{4.2}$ & 0.025 & 30.7 & $\overline{0.27}$ & $\overline{222}$ \\
\hline $\mathrm{CoTa}_{2}$ & 3.115 .2 & 0.31 & 2.613 .7 & 0.30 & 2.48 & 4.0 & 0.030 & 32.0 & 0.29 & 228 \\
\hline $\mathrm{NiW}_{2}$ & 3.024 .0 & 0.31 & 2.557 .6 & 0.34 & 2.38 & 4.3 & 0.041 & 37.7 & 0.31 & 294 \\
\hline $\mathrm{CoW}_{2}$ & 3.025 .1 & 0.37 & 2.546 .7 & 0.36 & 2.38 & 4.8 & 0.037 & 39.9 & 0.35 & 308 \\
\hline
\end{tabular}

TABLE IV: Data for tI12. R are average bond lengths for nearest neighbors under $4 \AA . k$ are averaged over atoms (i.e. weighted by the number of bonds per atom) in units $\mathrm{eV} / \AA^{2}, \rho_{\mathrm{COHP}}$ is iCOHP volume density in units $\mathrm{eV} / \AA^{3}$, and $K$ is the calculated bulk moduli in units GPa.

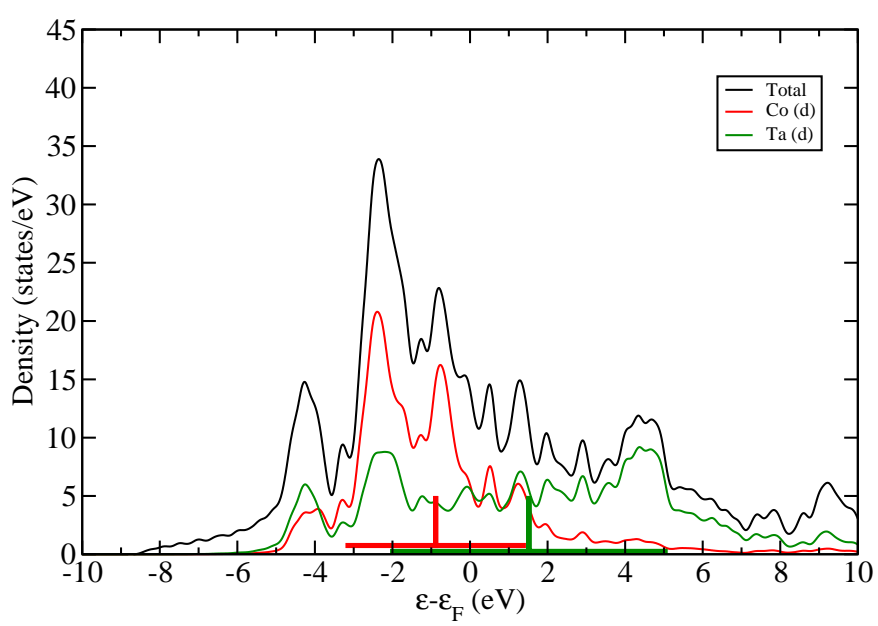

(a) CoTa.hR13

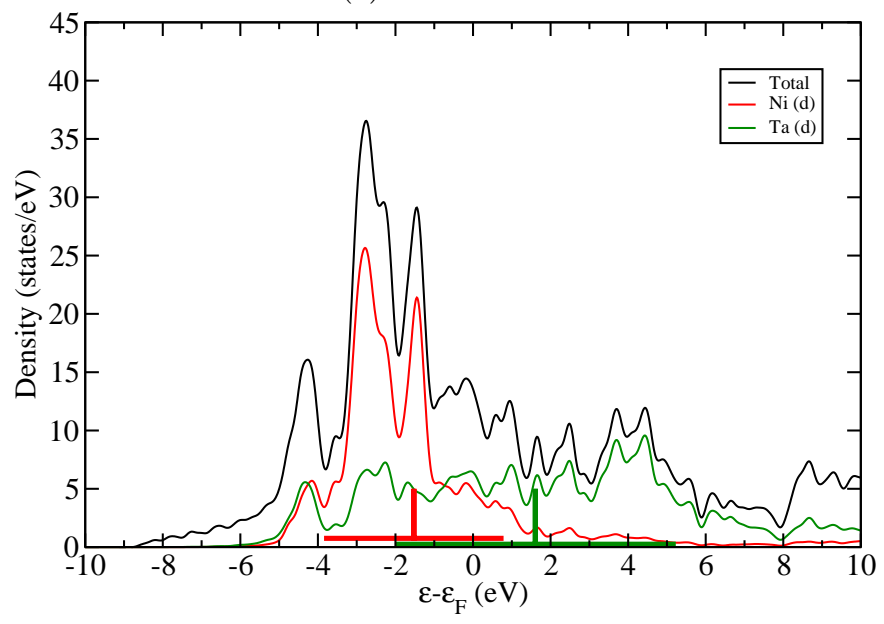

(c) NiTa.hR13

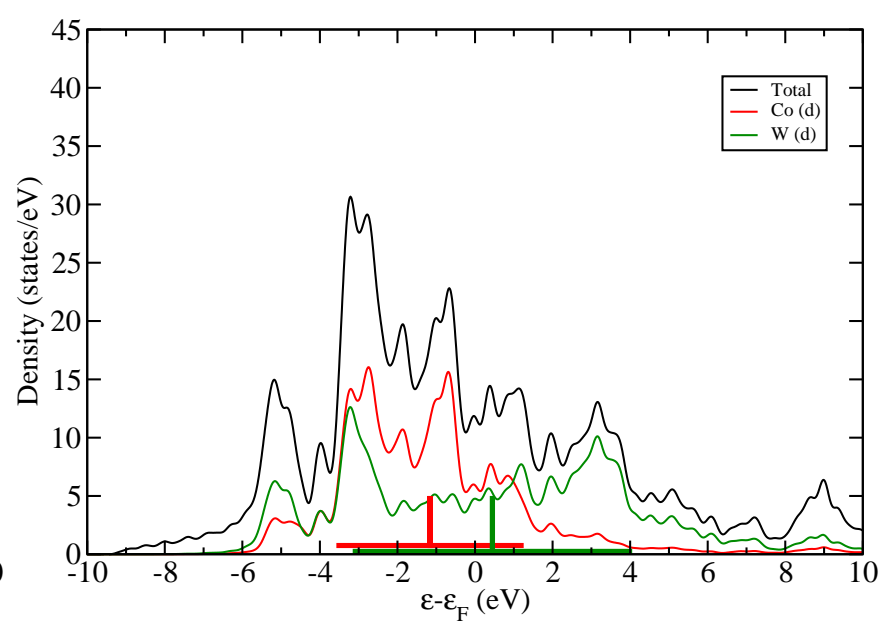

(b) CoW.hR13

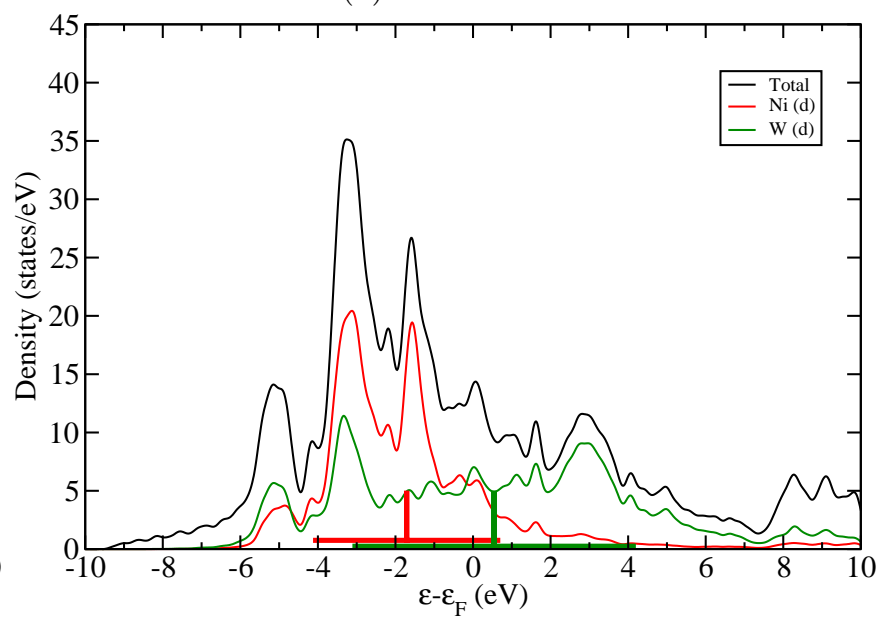

(d) NiW.hR13

FIG. 7: Electronic densities of states for hR13 structures. Line segments indicate the mean and standard deviations of the element projected $d$-bands.

(i.e. $\mathrm{AB}$ and $\mathrm{CD}$ ), binary enthalpies of formation for ETM-ETM and LTM-LTM families are weak compared to ETM-LTM families, suggesting that as a first approximation we may assume the differences in interspecies ETM-ETM and LTM-LTM bond strength average out.

Differences between our predicted interpolated elastic moduli and computed elastic moduli follow the trends previously reported for CPA. Again we see bulk moduli negligibly affected by atomic environment and pre- dominately determined by the alloying species, with deviations in bulk moduli below $2.6 \%$ for all structures. For shear moduli, the structures can be placed into two categories: those structures that have only one ETM species or else two ETM species from the same group (here $\mathrm{Nb}$ and $\mathrm{Ta}$ belong to group IV) which have deviations in shear moduli below $3.7 \%$, and those that have ETM species from different groups (here $\mathrm{W}$ from group $\mathrm{V}$ together with $\mathrm{Nb}$ or Ta from group IV) which have 


\begin{tabular}{l|lrr|lll} 
& & $\mathrm{K}$ & $\mathrm{G}$ & & $\mathrm{K}$ & $\mathrm{G}$ \\
\hline & $\mathrm{Co}$ & 218 & 107 & $\mathrm{Ni}$ & 200 & 93 \\
\hline $\mathrm{Nb}(\mathrm{K}=173, \mathrm{G}=22)$ & $\mathrm{Co}_{6} \mathrm{Nb}_{7}$ & 213 & 92 & $\mathrm{Ni}_{6} \mathrm{Nb}_{7}$ & 198 & 81 \\
$\mathrm{Ta}(\mathrm{K}=202, \mathrm{G}=61)$ & $\mathrm{Co}_{6} \mathrm{Ta}_{7}$ & 234 & 106 & $\mathrm{Ni}_{6} \mathrm{Ta}_{7}$ & 218 & 93 \\
$\mathrm{~W}(\mathrm{~K}=331, \mathrm{G}=143)$ & $\mathrm{Co}_{6} \mathrm{~W}_{7}$ & 302 & 117 & $\mathrm{Ni}_{6} \mathrm{~W}_{7}$ & 279 & 77
\end{tabular}

TABLE V: Elastic moduli for pure elements and binary hR13 structures.

\begin{tabular}{|c|c|c|c|}
\hline Chemical Formula & Calculated $\mathrm{K}, \mathrm{G}$ & Averaged $\mathrm{K}, \mathrm{G}$ & Deviation \\
\hline $\mathrm{Co}_{3} \mathrm{Ni}_{3} \mathrm{Nb}_{7}$ & 205,87 & 205,87 & $0.3,0.5$ \\
\hline $\mathrm{Co}_{3} \mathrm{Nb}_{3} \mathrm{Ta}_{4}$ & 225,100 & 223,99 & $0.7,1.4$ \\
\hline $\mathrm{Co}_{3} \mathrm{Ni}_{3} \mathrm{Ta}_{7}$ & 225,102 & 226,100 & $0.4,2.2$ \\
\hline $\mathrm{Ni}_{6} \mathrm{Nb}_{3} \mathrm{Ta}_{4}$ & 210,89 & 208,87 & $0.9,2.2$ \\
\hline $\mathrm{Co}_{3} \mathrm{Ni}_{3} \mathrm{~W}_{7}$ & 290,94 & 291,97 & $0.3,2.4$ \\
\hline $\mathrm{Co}_{3} \mathrm{Ni}_{3} \mathrm{Nb}_{3} \mathrm{Ta}_{4}$ & 217,97 & 216,93 & $0.1,3.7$ \\
\hline \hline $\mathrm{Co}_{6} \mathrm{Ta}_{4} \mathrm{~W}_{3}$ & 261,123 & 268,111 & $2.5,10.0$ \\
\hline $\mathrm{Co}_{3} \mathrm{Ni}_{3} \mathrm{Ta}_{3} \mathrm{~W}_{4}$ & 253,115 & 258,98 & $2.1,14.6$ \\
\hline $\mathrm{Co}_{6} \mathrm{Nb}_{3} \mathrm{~W}_{4}$ & 261,121 & 257,104 & $1.5,16.4$ \\
\hline $\mathrm{Ni}_{6} \mathrm{Nb}_{3} \mathrm{~W}_{4}$ & 241,92 & 239,79 & $0.8,16.8$ \\
\hline $\mathrm{Ni}_{6} \mathrm{Ta}_{4} \mathrm{~W}_{3}$ & 242,100 & 249,85 & $2.6,17.8$ \\
\hline $\mathrm{Co}_{3} \mathrm{Nb}_{3} \mathrm{Ni}_{3} \mathrm{~W}_{4}$ & 248,109 & 248,92 & $0.2,18.7$ \\
\hline
\end{tabular}

TABLE VI: Comparison of calculated ternary and quaternary hR13 moduli with averaged values of binaries. Moduli are given in GPa, and deviations in percentages.

deviations in shear moduli between $10.0 \%$ and $18.7 \%$. In all cases where predicted shear moduli deviate significantly from calculated shear moduli, the computed shear moduli have been enhanced.

That mixing $\mathrm{Co}$ and $\mathrm{Ni}$ or $\mathrm{Nb}$ and Ta causes little deviation in shear modulus, but mixing $\mathrm{Ta}$ and $\mathrm{W}$ does, is further evidence for the dependence of shear modulus on atomic environment. Co and $\mathrm{Ni}$ have similar atomic radii and electronegativity, as do $\mathrm{Nb}$ and $\mathrm{Ta}$. For a topologically close packed structure like hR13, substitution of these chemical species should not noticeably affect bond lengths and ionic charges, yielding similar calculated and averaged results. However, $\mathrm{Nb}$ and $\mathrm{Ta}$ have larger atomic radii and lower electronegativity than $\mathrm{W}$, leading to larger charge transfers and changes in bond length, reducing the accuracy of our averaging scheme while generally increasing bonding strength.

\section{CONCLUSIONS}

In this paper we examine the elasticity of various early transition metal-late transition metal crystalline binary alloys using first principles calculations and comparison with various averaging schemes. Calculated bulk moduli were reasonably close to the coherent potential approximation using pure elemental species, but CPA predictions were systematically low. This deviation correlates strongly with volume per atom. Larger and less regular deviations were observed for shear moduli, suggesting structural distortion being responsible for the deviation. Select ternary and quaternary structures were examined and confirmed these trends. To explain the dependence of elastic moduli on chemical bonding, force constants and electronic densities of state were calculated and it was found early transition metals are responsible for the strongest bonding, which agrees with observed trends in the bulk moduli. We find that Ni-W alloys have the largest Poisson ratios among the compositions studied and hence hold promise as the basis for design of ductile metallic glasses.

\section{ACKNOWLEDGEMENTS}

We would like to give special thanks to DOD-DTRA for funding this research under contract number DTRA11-1-0064.
1 T. Egami, Mat. Sci. Eng. A 2260228, 261 (1997).

2 T. Egami, S. J. Poon, Z. Zhang, and V. Keppens, Phys. Rev. B 76, 024203 (2007).

3 W. H. Wang, J. Appl. Phys. 99, 093506 (2006).

4 S. J. Poon, A. Zhu, and G. J. Shiflet, Appl. Phys. Lett. 92, 261902 (2008).

${ }^{5}$ H. S. Chen, J. T. Krause, and E. Coleman, J. Non-Cryst. Solids 18, 157 (1975).

6 J. J. Lewandowski, W. H. Wang, and A. L. Greer, Philos. Mag. Lett. 85, 77 (2005).

7 J. Q. Wang and H. Y. Bai, Scripta Mater. 61, 453 (2009).

8 W. L. Johnson and K. Samwer, Phys. Rev. Lett. 95, 195501 (2005).

${ }^{9}$ W. H. Wang, Prog. Mater. Sci. 57, 487 (2012).

10 F. C. Frank and J. S. Kasper, Acta Cryst. 11, 184 (1958).

11 F. C. Frank and J. S. Kasper, Acta Cryst. 12, 483 (1959).
12 A. K. Sinha, Topologically close-packed structures of transition metal alloys (Pergamon Press, 1972).

13 M. Telford, Materials Today 7, 36 (2004)

14 C. C. Hays, C. P. Kim, and W. L. Johnson, Phys. Rev. Lett. 84, 2901 (2000).

15 L. D. Landau and E. M. Lifshitz, Theory of Elasticity, 3rd ed., Course of Theoretical Physics, Vol. 7 (ButterworthHeinemann, 1986).

16 S. F. Pugh, Philos. Mag. 45, 823 (1954).

17 X. J. Gu, A. G. McDermott, S. J. Poon, and G. J. Shiflet, Appl. Phys. Lett. 88, 211905 (2006).

18 A. Inoue, B. Shen, H. Koshiba, H. Kato, and A. R. Yavari, Nature Mater. 2, 661 (2003).

19 M. Ohtsuki, R. Tamura, S. Takeuchi, S. Yoda, and T. Ohmura, Appl. Phys. Lett. 84, 4911 (2004).

20 J. F. Nye, Physical Properties of Crystals: Their Representation by Tensors and Matrices, 2nd ed. (Oxford Science 
Publications, 1985).

21 R. Zeller and P. H. Dederichs, Phys. Status Solidi B 55, 831 (1973).

${ }^{22}$ R. Hill, Proc. Phys. Soc. A 65, 349 (1952).

23 C. W. Nan, Prog. Mater. Sci. 37, 1 (1993).

24 Y. Zhang and A. L. Greer, J. Alloys Compd. 434-435, 2 (2007).

25 B. Zhang, R. J. Wang, D. Q. Zhao, M. X. Pan, and W. H. Wang, Phys. Rev. B 73, 092201 (2006).

${ }^{26}$ G. Kresse and J. Hafner, Phys. Rev. B 43, 558 (1993).

27 G. Kresse and J. Furthmüller, Phys. Rev. B 54, 11169 (1996).

28 P. E. Blöchl, Phys. Rev. B 50, 17953 (1994).

29 J. P. Perdew and Y. Wang, Phys. Rev. B 45, 13244 (1992).

30 M. Mihalkovic and M. Widom, Phys. Rev. B 70, 144107 (2004).

31 R. Dronskowski and P. E. Blöchl, J. Phys. Chem. 97, 8617 (1993).
32 O. Jepsen and O. K. Andersen, Z. Phys. B 97, 35 (1995).

33 A. Migliori and J. L. Sarrao, Resonant ultrasound spectroscopy (Wiley New York, 1997).

${ }^{34}$ H. W. Sheng, W. K. Luo, F. M. Alamgir, J. M. Bai, and E. Ma, Nature 439, 419 (2006).

35 X. W. Fang, C. Z. Wang, S. G. Hao, M. J. Kramer, Y. X. Yao, M. I. Mendelev, Z. J. Ding, R. E. Napolitano, and K. M. Ho, Scientific Reports 1, 194 (2011).

36 J. D. Bernal, Proc. R. Soc. Lond. A 280, 299 (1964).

37 D. R. Nelson, Phys. Rev. B 28, 5515 (1983).

38 D. B. Miracle, JOM 64, 846 (2012).

39 J. D. Honeycutt and H. C. Andersen, J. Phys. Chem. 91, 4950 (1987).

40 P. Ganesh and M. Widom, Phys. Rev. B 77, 014205 (2008).

41 J. M. Walsh and J. M. J. Donachie, Met. Trans. 4, 2854 (1973).

42 D. Tromans, IJRRAS 6, 462 (2011).

${ }^{43}$ X. F. Liu, R. J. Wang, and W. H. Wang, Scripta Mater. 62, 254 (2010). 